\title{
Fine Needle Aspiration Cytology in the Diagnosis of Intraabdominal Malignancies
}

\author{
Prabhakar Ulaganathan \\ Senior Civil Surgeon, Department of surgery, Government District Head Quarters Hospital, Kumbakonam, India
}

Corresponding author: Dr Prabhakar Ulaganathan, 70, Jagannatha Pillayar Koil West Street, Kumbakonam, Tamilnadu, India

DOI: http://dx.doi.org/10.21276/ijcmsr.2020.5.2.22

How to cite this article: Prabhakar Ulaganathan. Fine needle aspiration cytology in the diagnosis of intraabdominal malignancies. International Journal of Contemporary Medicine Surgery and Radiology. 2020;5(2):B88-B93.

\section{A B S T R A C T}

Introduction: The pathological diagnosis of the intraabdominal malignancy is arrived by any one of the biopsy techniques. Such techniques have many disadvantages. FNAC of the intra abdominal malignancy is often a forgotten rather than neglected investigation. But it can be done with confidence to arrive at the tissue diagnosis. Aim: To emphasize FNAC as a sensitive and specific diagnostic tool for intra abdominal malignancies.

Material and methods: FNAC was done in 50 cases of palpable intraabdominal malignancies and compared with other conventional biopsy techniques.

Results: FNAC gave positive report of malignancy in $80 \%$ of cases, specifying the type and differentiation in most cases. FNAC of intra abdominal malignancies had a sensitivity of $86.96 \%$ and a specificity of $100 \%$.The overall accuracy rate is $88 \%$. There were no false positive reports. It had no complications. It reduced cost and time for investigations.

Conclusion: FNAC was extremely helpful in arriving at a cytological diagnosis of palpable intraabdominal malignancy. This was useful in the further management of the patients. FNAC can be safely used as first line investigation for intraabdominal malignancies.

Keywords: FNAC, Biopsy, Fine Needle, Cytodiagnosis, Neoplasms, Diagnostic Tool, Abdominal Cavity

\section{INTRODUCTION}

Intra abdominal malignancy is one of the commonest malignancies treated in any institution. It can arise from any intra abdominal organ of gastrointestinal system, hepatobiliary system, urogenital system or retroperitoneum. The pathological diagnosis is usually arrived by any one of the biopsy techniques such as upper gastrointestinal endoscopy, sigmoidoscopy, colonoscopy, trucut biopsy or by laparotomy and open biopsy technique. Such investigations are costly and time consuming with morbidity and mortality. Sometimes open biopsy of an advanced intra-abdominal malignancy may increase the morbidity and mortality with poor results. But FNAC can be used to arrive at the pathological diagnosis.

KUN did first the Fine needle aspiration cytology in 1847. Since then, FNAC has undergone gradual improvement and has become increasingly popular. FNAC was first tried in Sweden and Denmark for intraabdominal masses. ${ }^{1}$ Since then various studies were conducted regarding the use of FNAC in the diagnosis of intraabdominal malignancies either with or without guidance for various intraabdominal malignancies. $^{2-5}$ These studies showed high success rate and low complications rate.

FNAC is a safe, quick and accurate method with low morbidity. The acceptance rate is high by the patients. This study was done without using any imaging techniques with a view to demonstrate the usefulness of FNAC in various intraabdominal malignancies and commend its wider use as an important tool in the initial assessment for any intraabdominal malignancies.

\section{MATERIAL AND METHODS}

This study was conducted in the Thanjavur Medical college Hospital, Thanjavur from May 1999 to October 2000. 50 patients of palpable abdominal mass with a clinical diagnosis of malignancy were selected for the study. Patients without palpable mass and patients having bleeding diathesis were excluded from the study. FNAC was done percutaneously without any radiological or sonological guidance and sent for cytological examination.

50 patients underwent the study (Table no1). Of them 39 patients were males and 11 were females. Thorough clinical examination and necessary investigations were done (Tables no 2\&3).

21 patients had a clinical diagnosis of Carcinoma stomach, of them 17 patients were males and 4 were females. Patients were aged from 38-71years, with most of the patients being aged between 50-60 years. FNAC was done for all the 21 cases of Carcinoma Stomach.

14 patients had a clinical diagnosis of colonic malignancy and underwent FNAC. Of them 12 patients were males and 2 were females. They were aged between 29 and 80 years, with most of the patients in the 50-60 age groups. 9 patients had right iliac fossa mass. 
10 patients who had palpable liver mass were included in the study and underwent FNAC. Of them 8 patients were males and 2 were females. Patients were aged from 40 to 70 years, with most of the patients aged between 40 to 50 years. Of them 5 were cases of hepatocellular carcinoma, 4 were cases of secondary adenocarcinomatous deposits and one was a case of chronic liver abscess.

3 patients who had retro-peritoneal masses were subjected to FNAC. Of them 2 patients were females and 1 was male. 1 patient was aged 52 years and 2 patients were aged 60 and 65 years.

A 56 year old male presented with complaints of obstructive jaundice and right hypochondrial mass. A clinical diagnosis of carcinoma of the head of pancreas was made and FNAC was done.

A female patient aged 58 years presented with right hypochondrial mass. A clinical diagnosis of carcinoma of the gal1 bladder was made. FNAC was done.

Two slides are prepared for each case. Adequate samples for cytological interpretation were obtained in all cases. Hematoxylin and Eosin staining of slides was done. Slides were examined by pathologist for reporting. Distinctly palpable masses were biopsied in the outpatient clinic to save time and expense. In our study since all the cases had distinctly palpable abdominal mass; the procedure was done percutaneously without any guidance. There were no complications.

Ultrasound abdomen, upper G.I. endoscopy, sigmoidoscopy, colonoscopy, Barium study, Intra venous urography and CT scan abdomen were done to know the origin and extension of the lesions. For evaluation of sensitivity and specificity of FNAC other biopsy techniques were undertaken along with it.

\section{RESULTS}

For 21 cases of Carcinoma Stomach, FNAC was positive for Malignancy in 17 patients and reported as adenocarcinoma. Histological subtypes and grading were also reported by FNAC (Figures 1\&2). 4 cases were negative for Malignancy by FNAC. Upper gastro-intestinal endoscopy was done for all the 21 cases; all patients had growth in the stomach.

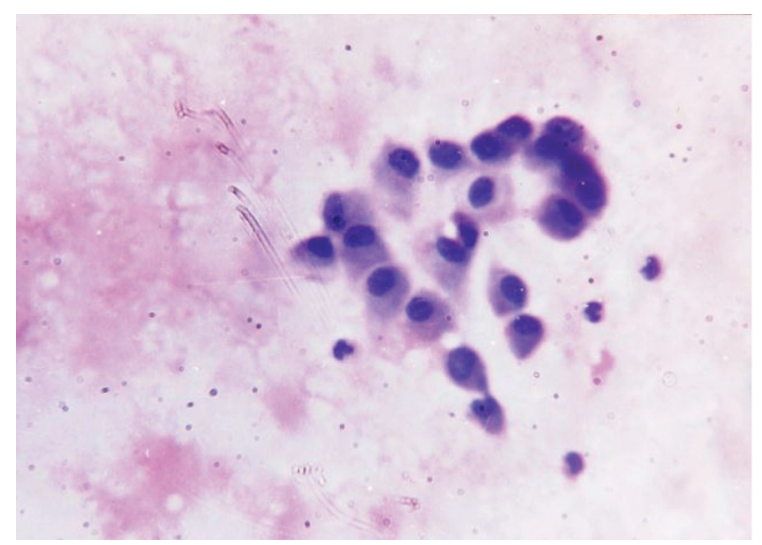

Figure-1: Adenocarcinoma stomach

Malignant cuboidal epithelial cells with pleomorphic nuclei and moderate eosinophilic cytoplasm arranged in a glandular pattern suggestive of adenocarcinoma stomach
Biopsy was taken and sent for histopathological examination. Malignancy was diagnosed in all the cases including the 4

\begin{tabular}{|l|c|}
\hline Diagnosis & No of cases \\
\hline Carcinoma stomach & 21 \\
\hline Colonic malignancy & 14 \\
\hline Liver mass & 10 \\
\hline Retroperitoneal mass & 3 \\
\hline Carcinoma pancreas & 1 \\
\hline Carcinoma gall bladder & 1 \\
\hline Total & 50 \\
\hline \multicolumn{2}{|c|}{ Table-1: Distribution of cases } \\
\hline
\end{tabular}

\begin{tabular}{|l|c|}
\hline Symptoms & No of patients \\
\hline Abdominal pain & 50 \\
\hline Abdominal mass & 50 \\
\hline Weight loss & 48 \\
\hline Anorexia & 40 \\
\hline Melaena & 15 \\
\hline Hemetemesis & 1 \\
\hline Vomiting & 9 \\
\hline Dysphagia & 1 \\
\hline Altered bowel habits & 11 \\
\hline Bleeding PR & 3 \\
\hline Clay coloured stools & 2 \\
\hline Jaundice & 6 \\
\hline Abdominal distension & 4 \\
\hline Fever & 1 \\
\hline Pruritis Table-2: Patient symptoms \\
\hline \multicolumn{2}{|c|}{2} \\
\hline
\end{tabular}

\begin{tabular}{|l|c|}
\hline Signs & No of patients \\
\hline Abdominal mass & 50 \\
\hline Anemia & 41 \\
\hline Jaundice & 6 \\
\hline Cachexia & 8 \\
\hline Pedal edema & 3 \\
\hline Ascites & 4 \\
\hline \multicolumn{2}{|c|}{ Table-3: Patient signs } \\
\hline
\end{tabular}

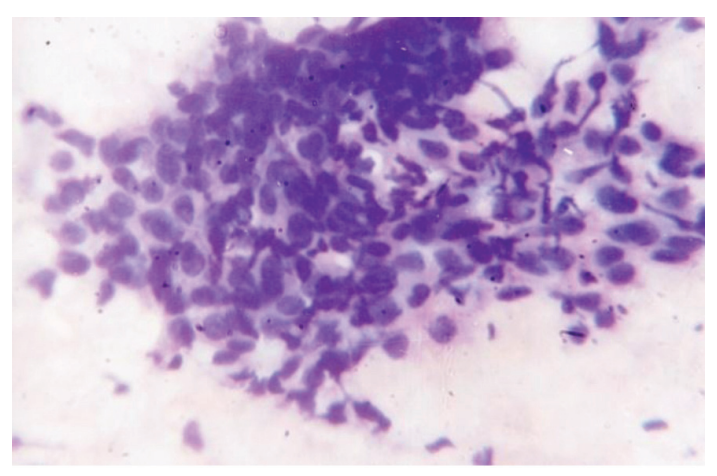

Figure-2: Poorly differentiated adenocarcinoma of stomach Papillary clusters of malignant epithelial cells with hyperchromatic pleomorphic nuclei. Lower down it shows a tight cluster in between polymorphic cells Nucleoli are not prominent. Some areas show glandular pattern indicating a poorly differentiated adenocarcinoma of stomach. 
cases which were negative by FNAC. We encountered technical problems in 2 Patients due to obesity. Of 21 patients of carcinoma stomach, FNAC was positive in 17 patients and negative in 4 patients.

FNAC was done for 14 patients of colonic malignancy. 9 patients had right iliac fossa mass. FNAC was positive as adenocarcinoma in 6 patients. Colonoscopy found the growth in 6 patients; biopsy was taken and sent for histopathological examination. Of them 5 were cases of adenocarcinoma of the ascending colon and one were a case of adenocarcinoma cecum. The aspirate in three cases showed inflammatory cells. Of them, 2 patients had mantoux and other tests positive for tuberculosis. FNAC was positive as adenocarcinoma in 2 cases of hepatic flexure growth and one case each of transverse colon growth, carcinoma descending colon and carcinoma sigmoid colon. All these patients were confirmed by colonoscopy and biopsy. Of 14 cases of colonic malignancy, FNAC was positive in 11 cases and negative in 3 cases (Figure no 3).

FNAC was done for 10 patients with palpable liver mass. Of them 5 were cases of hepatocellular carcinoma, 4 were cases of secondary adenocarcinomatous deposits and one was a case of chronic liver abscess. FNAC was positive for malignancy in 7 patients and negative in 3 patients. FNAC was positive in 4 cases of hepatocellular carcinoma

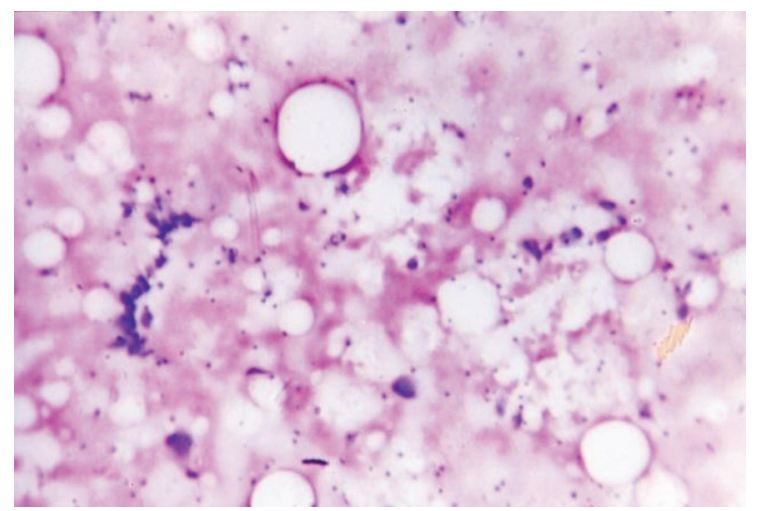

Figure-3: Mucin secreting adenocarcinoma of colon

Strips of malignant epithelial cells surrounded by mucin secreting cells suggestive of mucin secreting adenocarcinoma of colon

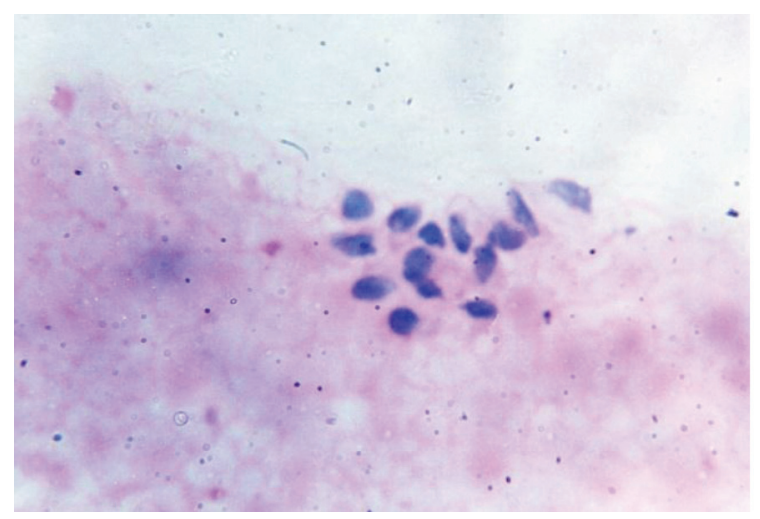

Figure-4: Hepatocellular carcinoma

Linear strips and clusters of malignant cells with angulated nuclei, prominent nucleolus and moderate pink cytoplasm suggestive of hepatocellular carcinoma and 3 cases of secondary adenocarcinomatous deposits (Figures no 4\&5). Trucut biopsy was done for all the 10 cases. Trucut biopsy was positive for malignancy in 9 cases including 5 cases of hepatocellular carcinoma and 4 cases of secondary adenocarcinomatous deposits. 7 out of the 10 cases of liver mass were diagnosed as malignant by FNAC.

FNAC was positive for malignancy in all the cases as 3 cases of retroperitoneal masses as secondary adenocarcinomatous deposits (figure no 6).

FNAC was done for a case of carcinoma of the head of pancreas. The report came as adenocarcinoma, which was confirmed after open biopsy (figure no7).

FNAC was done for a case of carcinoma of gall bladder. The report came as adenocarcinoma, which was confirmed after open biopsy (figure no 8).

The results of FNAC done for 50 patients was as follows, 40 results were true positive, false negative results in 6 cases, true negative results in 4 cases. There was no false positive result.

To summarise in this study FNAC had the sensitivity of $86.96 \%$, specificity of $100 \%$, false negative rate of $13.04 \%$ and nil false positive rate. It had $100 \%$ positive predictive value and $40 \%$ negative predictive value. The overall accuracy rate is $88 \%$.

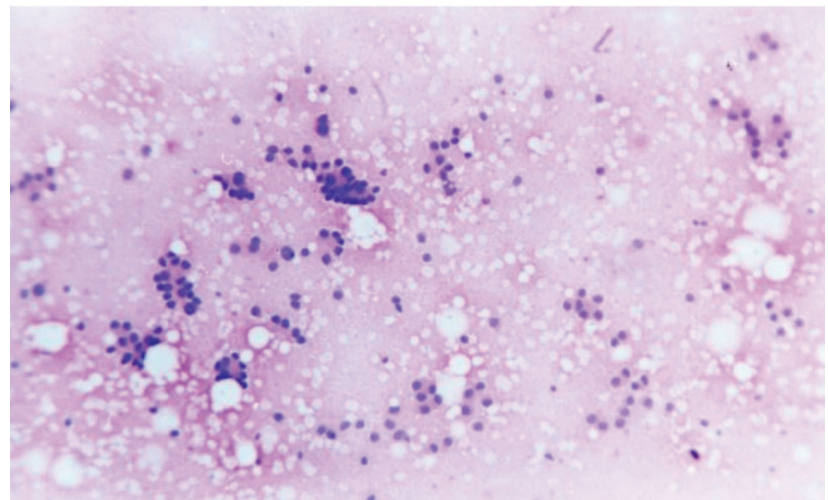

Figure-5: secondary adenocarcinoma deposits of liver Small islands of malignant cells arranged in a glandular pattern in the case of adenocarcinoma deposits in liver.

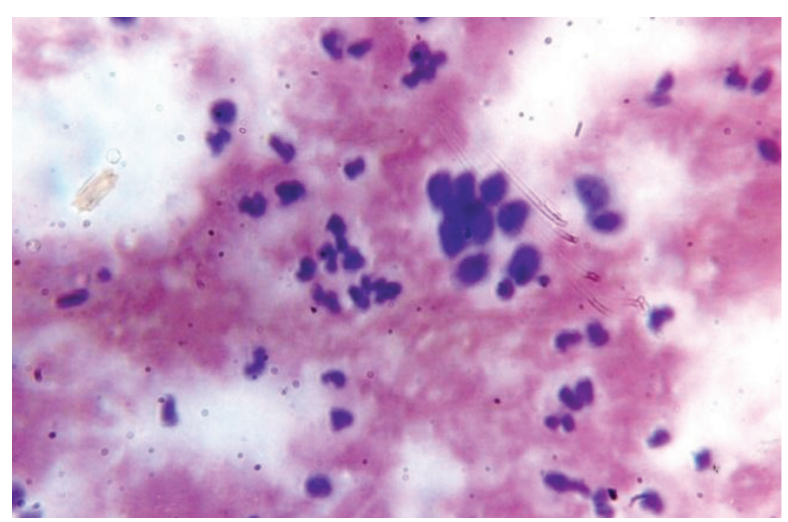

Figure-6: Retroperitoneal metastatic adenocarcinoma

Poorly differentiated malignant epithelial cells with necrotic inflammatory background in a case of retroperitoneal metastatic adenocarcinoma 


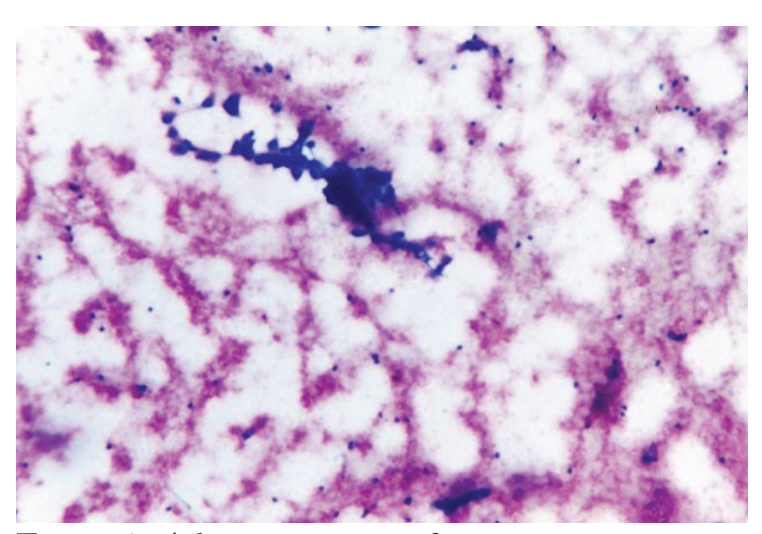

Figure-7: Adenocarcinoma of pancreas

Clusters of tiny malignant epithelial cells in a hemorrhagic background with minimal cytoplasm suggestive of adenocarcinoma of pancreas

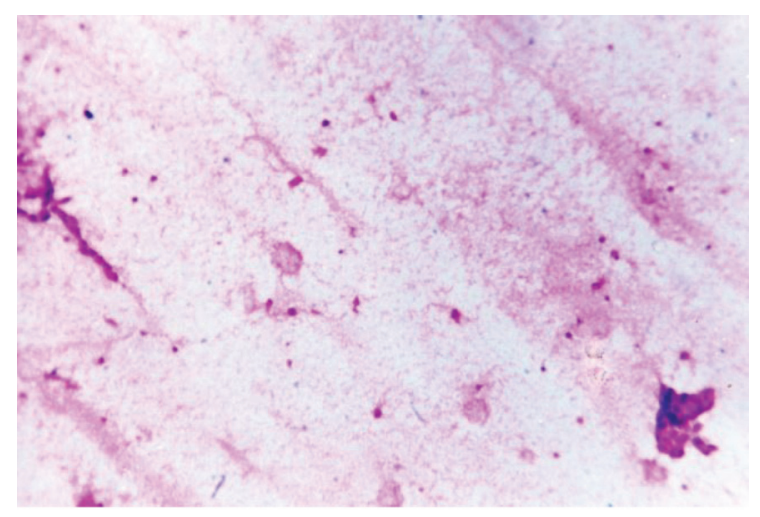

Figure-8: Adenocarcinoma of Gall bladder

Clusters of tiny malignant epithelial cells in a hemorrhagic background with minimal cytoplasm suggestive of adenocarcinoma of Gallbladder

\section{DISCUSSION}

Diagnosis of the gastrointestinal tract tumours is usually done by endoscopic biopsy technique. FNAC can sometimes be used as first line investigation of a palpable abdominal mass. Adenocarcinoma of the gastro intestinal tract may be well or poorly differentiated and more or less mucin secreting. A poorly differentiated adenocarcinoma of signet ring cells with intra cytoplasmic mucin vacuoles is most likely of gastric origin. A well differentiated adenocarcinoma of columnar cells showing a palisade arrangement and with tumour necrosis is probably of Colonic origin. We had no complications following FNAC of such lesions. The FNAC in obstructed and distended bowel should be avoided. ${ }^{6}$ In the present study 17 out of the 21 cases of carcinoma stomach and all cases of colonic malignancies are diagnosed by FNAC. Malignancy in the liver when presents late and clinically inoperable needs less invasive investigations to confirm the diagnosis. FNAC is useful in that situation. FNAC has now largely replaced the conventional large needle core biopsy in the diagnosis of focal lesions. The 22-23 gauge fine needles have less discomfort and a very low risk of complications. Hospitalisation of the patient is not necessary. The accuracy of large core needle biopsy is quite variable between 60 $82 \%$ in the literature. ${ }^{6}$ The diagnostic sensitivity is reported around $90 \%$ or even higher. ${ }^{7,8}$ In the present study 7 out of the 10 cases were reported positive.

In the case of retroperitoneal tumours, FNAC can be done in addition to other investigations without much risk and cost. It may be considered in advanced inoperable tumours when pre operative embolisation or irradiation is planned. ${ }^{9}$ Palliative radiotherapy or chemotherapy can be started in advanced inoperable cases of metastatic tumours and malignant lymphoma after FNAC. In retroperitoneal lymphadenopathy FNAC is a convenient method to confirm metastatic involvement or to differentiate between metastatic malignancy and malignant lymphoma. ${ }^{10}$ FNAC of lymphangiographically abnormal retro peritoneal lymph nodes has an average success rate of $70 \%$ in obtaining a representative sample from an individual node. Overall accuracy was $83 \% .{ }^{11,12}$ The diagnosis of primary soft tissue tumours of the retroperitoneum by FNAC is not possible. In the present study 3 cases of retroperitoneal secondary deposits were reported.

FNAC of a pancreatic mass sometimes used to confirm inoperable malignancy so that the patient may be spared of a mere explorative diagnostic laparotomy. Pancreatic malignancy can diagnosed with $100 \%$ specificity. ${ }^{13-15}$ Diagnostic sensitivity is much more variable between 50$90 \%$ even with guided FNAC procedures. In our study one case of pancreatic carcinoma was reported.

Diagnostic accuracy tends to be lower for biliary tumours prompting the search for reliable endoscopic FNAC techniques. ${ }^{16}$ In the present study one case of carcinoma gall bladder was reported.

No complications had occurred in the present study. In the literature also the complications are minimal and rarely reported. When stomach or colon is biopsied with 22 gauges or less size thin needles the complications are minimal. Lundquist in 2611 FNACs of liver had reported only one intra hepatic hematoma as significant complication without any mortality. ${ }^{17}$ Isolated cases of severe or fatal haemorrhage two cases each of bile peritonitis and of seeding in the needle track have been reported. Cases of needle track seeding were reported in cases, when needle size is thicker than 21 gauge was used for FNAC. ${ }^{18}$ Other rare complications like carcinoid crisis, lymphorrhea and pneumothorax were reported. A few examples of severe pancreatitis or exacerbation of pancreatitis have been recorded following FNAC of the pancreas. ${ }^{19} \mathrm{~A}$ single case of septicaemia following pancreatic biopsy had been reported. Needle track tumour seeding following FNAC of the pancreatic carcinoma had been reported. ${ }^{20}$ Handling of pancreatic tumour which had resulted in ascites in two cases was reported. ${ }^{21}$ In solid renal tumours complications were recorded in $0.4 \%$ of cases. ${ }^{22,23} \mathrm{~A}$ small number of cases of needle tract implantation after FNAC of renal cancer of transitional cell carcinoma and of an adrenal tumour have been reported. ${ }^{24} \mathrm{~A}$ case of hypertensive crisis after FNAC of adrenal pheochromocytoma was reported. ${ }^{25}$

Contraindications are few for this procedure. FNAC is contraindicated in Aortic aneurysm. An aneurysm may not pulsate if it is filled with thrombus and appear solid in ultrasonogram. FNAC is contraindicated in bleeding disorders. Routine checking of prothrombin time and the 
platelet count should be done before doing FNAC. Previously Hydatid cysts were relative contra indication for FNAC. No allergic reactions or anaphylaxis have been observed in two reported case series of accidental aspiration of hydatid cysts. A case of anaphylactic shock after aspiration of hydatid cyst with 18 gauge needle was reported. ${ }^{26}$ Hemangioma is no longer considered as contraindication to FNAC. The correct less traumatic technique has to be followed to avoid haemorrhage. ${ }^{27}$ Adrenal pheochromocytoma is not an absolute contraindication for FNAC when necessary precautions are taken to deal with any complication. ${ }^{25}$

In 1984, Bree R L have done the study of 117 patients who had intra-abdominal mass, for whom FNAC was done with computed tomography and ultrasound guidance had an accuracy rate of $86 \% .^{2}$

In 1989, Jan G M and Mahajan R have done a study of ultrasound guidance fine needle aspiration biopsy in 178 patients with clinically suspected intra abdominal and retroperitoneal masses. They reported an accuracy rate of $70 \% .^{3}$

Khan A et al.1996 had reported an accuracy rate of $97.33 \%$ in 50 cases of palpable abdominal masses. Fine needle aspirates were positive in 44 out of 45 malignant lesions. No false positive results were obtained. There were no complications. ${ }^{4}$ In 1998, Muzaffar Ahmad Zargar had reported 87.5\% accuracy. In his study of 50 cases adequate cytological material was obtained in 45 cases. In 40 cases which proved malignant, 35 were correctly diagnosed by FNAC with accuracy rate of $87.5 \% .^{5}$

In the present study the accuracy rate was $88 \%$.

\section{CONCLUSION}

In this study of 50 cases, FNAC gave positive report of malignancy in 40 cases (80\%), specifying the type and differentiation of malignancy. Except for a mild local discomfort, there were no complications. There were no false positive reports. So FNAC can be done as an initial diagnostic procedure for any intraabdominal malignancy.

\section{ACKNOWLEDGEMENTS}

I thank my unit Chief Prof. S. Narendran MS., Ph.D., our unit assistant Professors Dr. G. Ambujam M.S., Dr.T.Anantharamakrishnan M.S., Prof. Dr. V.Banumathy M.D., Professor of Pathology for their constant guidance and encouragement throughout the period of this study and without whose help this study would not have been possible.

\section{REFERENCES}

1. Oscarson J. Selective angiography in fine-needle aspiration cytodiagnosis of gastric and pancreatic tumours. 1972;

2. Bree RL, Jafri SZH, Schwab RE, Farah J, Bernacki EG, Ellwood RA. Abdominal fine needle aspiration biopsies with CT and ultrasound guidance: techniques, results and clinical implications. Comput Radiol. 1984;8(1):915.

3. Jan GM, Mahajan R. Ultrasound guided percutaneous fine needle aspiration biopsy (FNAB) of intraabdominal and retroperitoneal masses. Indian J Gastroenterol.
1989;8(2):99-100.

4. Khan AA, Jan GM, Wani NA. Fine needle aspiration of intra-abdominal masses for cytodiagnosis. J Indian Med Assoc. 1996;94(5):167-8.

5. Muzaffar ahmad zargar, Sabina magbool Arshad shafi. fine needle aspiration as an initial tool in evaluation of abdominal masses. Indian J Surg. 1998;60(3).

6. Orell SR, Sterrett GF, Walters M-I, Whitaker D. Manual and atlas of fine needle aspiration cytology. Churchill Livingstone; 1999.

7. Babb RR, Jackman RJ. Needle biopsy of the liver. A critique of four currently available methods. West J Med. 1989;150(1):39.

8. Jacobsen GK, Gammelgaard J, Fuglø M. Coarse needle biopsy versus fine needle aspiration biopsy in the diagnosis of focal lesions of the liver. Ultrasonically guided needle biopsy in suspected hepatic malignancy. Acta Cytol. 1983;27(2):152-6.

9. Niceforo J, Coughlin BF. Diagnosis of renal cell carcinoma: value of fine-needle aspiration cytology in patients with metastases or contraindications to nephrectomy.AJR Am J Roentgenol.1993;161(6):13035.

10. Al-Mofleh IA.Ultrasound-guided fine needle aspiration of retroperitoneal, abdominal and pelvic lymph nodes. Diagnostic reliability. Acta Cytol. 1992;36(3):413-5.

11. Wajsman Z, Gamarra M, Park JJ, Beckley S, Pontes JE. Transabdominal fine needle aspiration of retroperitoneal lymph nodes in staging of genitourinary tract cancer (correlation with lymphography and lymph node dissection findings). J Urol. 1982;128(6):1238-40.

12. Rupp N, Rothenberger KH. Transabdominal Lymph Node Biopsy. In: Percutaneous Biopsy and Therapeutic Vascular Occlusion: International Symposium, München 1979. Thieme; 1980. p. 70.

13. Mitchell ML, Bittner CA, Wills JS, Parker FP. Fine needle aspiration cytology of the pancreas. A retrospective study of 73 cases. Acta Cytol. 1988;32(4):447-51.

14. Søreide O, Skaarland E, Pedersen OM, Larssen TB, Arnesjø B. Fine-needle biopsy of the pancreas: results of 204 routinely performed biopsies in 190 patients. World J Surg. 1985;9(6):960-4.

15. Jorda M, Essenfeld H, Garcia E, Ganjei P. The value of fine needle aspiration cytology in the diagnosis of inflammatory pancreatic masses. Diagn Cytopathol. 1992;8(1):65-7.

16. Hall-Craggs MA, Lees WR. Fine-needle aspiration biopsy: pancreatic and biliary tumors. Am J Roentgenol. 1986;147(2):399-403.

17. Lundquist A. Liver biopsy with a needle of $0.7 \mathrm{~mm}$ outer diameter: safety and quantitative yield. Acta Med Scand. 1970;188(16):471-4.

18. Nguyen GK. Fine needle aspiration biopsy of hepatic tumours in adults. In: Sommers SC, Rose P.P FRE, editor. pathology annals. appleton \& lange; 1986. p. 321-49.

19. Mueller PR, Miketic LM, Simeone JF, Silverman SG, Saini S, WittenbergJ, et al. Severe acute pancreatitis after percutaneous biopsy of the pancreas. Am J Roentgenol. 1988;151(3):493-4.

20. Bergenfeldt M, Genell S, Lindholm K, Ekberg O, 
Aspelin P. Needle-tract seeding after percutaneous fine-needle biopsy of pancreatic carcinoma. Case report. Acta Chir Scand. 1988;154(1):77-9.

21. Rosenbaum DA, Frost DB. Fine needle aspiration biopsy of the pancreas complicated by pancreatic ascites. Cancer. 1990;65(11):2537-8.

22. Orell SR. The diagnosis of solid renal and adrenal masses by aspiration cytology. In: Aspiration Cytology in the Staging of Urological Cancer. Springer; 1988. p. 215-23.

23. Pilotti S, Rilke F, Alasio L, Garbagnati F. The role of fine needle aspiration in the assessment of renal masses. Acta Cytol. 1988;32(1):1-10.

24. Wehle MJ, Grabstald H. Contraindications to needle aspiration of a solid renal mass: tumor dissemination by renal needle aspiration. J Urol. 1986;136(2):446-8.

25. Casola G, Nicolet V, VanSonnenberg E, Withers C, Bretagnolle M, Saba RM, et al. Unsuspected pheochromocytoma: risk of blood-pressure alterations during percutaneous adrenal biopsy. Radiology. 1986;159(3):733-5.

26. Das DK, Bhambhani S, Pant CS. Ultrasound guided fine needle aspiration cytology: Diagnosis of hydatid disease of the abdomen and thorax. Diagn Cytopathol. 1995;12(2):173-6.

27. Caturelli E, Rapaccini GL, Sabelli C, De Simone F, Fabiano A, Romagna Manoja E, et al. Ultrasound guided fine needle aspiration biopsy in the diagnosis of hepatic hemangioma. Liver. 1986;6(6):326-30

Source of Support: Nil; Conflict of Interest: None

Submitted: 18-04-2020; Accepted: 30-05-2020; Published online: 26-06-2020 